\title{
ULTRAPOROUS NITROGEN-DOPED ZEOLITE-TEMPLATED CARBON FOR HIGH POWER DENSITY AQUEOUS-BASED SUPERCAPACITORS
}

\author{
María José Mostazo-López ${ }^{1}$, Ramiro Ruiz-Rosas ${ }^{1}$, Alberto Castro-Muñiz², Hirotomo \\ Nishihara $^{2}$, Takashi Kyotani ${ }^{2}$, Emilia Morallón ${ }^{3}$, Diego Cazorla-Amorós ${ }^{1}$ \\ ${ }^{1}$ Departamento de Química Inorgánica e Instituto Universitario de Materiales, Universidad de \\ Alicante, Apartado 99, 03080-Alicante, España \\ ${ }^{2}$ Institute of Multidisciplinary Research for Advanced Materials, Tohoku University, 2-1-1 \\ Katahira, Aoba, Sendai, Japan \\ ${ }^{3}$ Departamento de Química Física e Instituto Universitario de Materiales, Universidad de Alicante, \\ Apartado 99, 03080-Alicante, España
}




\begin{abstract}
Two zeolite templated carbons (ZTC) with comparable structure and different surface chemistry have been synthesized by chemical vapor deposition of different precursors, producing a non-doped and a $\mathrm{N}$-doped carbon material (4 at. \% XPS) in which most of the functionalities are quaternary N.

A larger specific capacitance (farads per surface area) has been measured in acid electrolyte for the $\mathrm{N}$-doped ZTC, that can be related to an improved wettability due to the presence of nitrogen and oxygen. The capacitance of N-doped ZTC is lower in alkaline electrolyte, probably due to the loss of electrochemical activity of certain oxygen functionalities. Interestingly, the electro-oxidation process of N-ZTC implies lower irreversible currents (providing higher electrochemical stability) than for ZTC. The presence of quaternary nitrogen greatly improves the electric conductivity of N-ZTC, which shows a superior rate performance.

ZTC and N-ZTC capacitors were constructed using $1 \mathrm{M} \mathrm{H}_{2} \mathrm{SO}_{4}$. Under the same conditions, N-doped ZTC based capacitor has higher energy density, 6.7 vs $5.9 \mathrm{~W} \mathrm{~h} / \mathrm{kg}$. The power density of N-ZTC is four times higher, producing an outstanding maximum power of $98 \mathrm{~kW} / \mathrm{kg}$. These results provide clear evidences of the advantages of doping advanced porous carbon materials with nitrogen functionalities.
\end{abstract}




\section{INTRODUCTION}

Supercapacitors are one of the most relevant energy storage devices due to their extraordinary power density, since this is much higher than that provided by other systems, such as batteries and fuel cells. These devices can be composed by different electrode materials (carbon materials, conducting polymers and metal oxides) and electrolytes (aqueous, organic and ionic liquids). However, the development of the greener, safer and cheaper aqueous-based supercapacitors is severely limited by their low operation voltage. In consequence, their energy density is lower than that showed by those supercapacitors based on organic or ionic liquid based electrolytes [1-3].

Most of the strategies for overcoming this limitation are focused on the development of electrode materials with high electrochemical stability and capacitance. Among all the possibilities, the development of ultraporous carbon materials with well-developed microporosity and ordered structure is highly desirable. In this sense, zeolite-templated carbons (ZTC) evidence a unique 3Dordered microporous structure that shows extremely profitable properties for this application $[4,5]$. First, they have an outstanding apparent surface area and an ordered structure with uniform pore size distribution $(1.2 \mathrm{~nm})$ that maximizes the formation of the electrical double layer and improves the mobility of ions within the porosity, making them especially interesting as electrode materials for supercapacitors. Moreover, a large amount of highly reactive edge sites are present in their structure, which eases the incorporation of electroactive oxygen functionalities that produce an extraordinary pseudocapacitance boost in acid media [6]. However, these materials can be easily overoxidized, leading to the degradation of the structure and the loss of their unique properties.

The electrochemical performance of carbon materials can be tuned by the introduction of heteroatoms (such as $\mathrm{O}, \mathrm{N}, \mathrm{P}$ or B) [7-16]. Concretely, nitrogen functional groups have been found to modify the electrochemical stability, electrical conductivity, the wettability and pseudocapacitance of carbon materials $[8,17-20]$. Nevertheless, the role of the different $\mathrm{N}$ functionalities still is not fully understood. Nitrogen-doped carbons can be synthesized following two different strategies: (i) by using a nitrogen-containing precursor as source or (ii) through post-treatments of a carbon material with nitrogen reagents [21,22]. However, these approaches usually require the use of high temperatures that would damage the fragile porous network of ZTCs. Hence, a plausible strategy for obtaining N-doped ZTC while preserving its unique structure would rely on the use of a nitrogencontaining gas as a raw precursor. Following this premise, Kyotani et al [7,23,24] synthesized N- 
ZTC by using acetonitrile as CVD precursor. By careful combination of the CVD conditions and the template, they were able to obtain a nitrogen-rich microporous ZTC with a similar structure of nondoped ZTC, while showing a larger microporosity than that found for other N-doped ZTCs in the literature [25,26]. The advantageous ordered structure of the resulting material was profited for achieving a better understanding of the effect of $\mathrm{N}$-doping in the electrochemical performance of carbon materials in organic electrolyte [27], allowing to use them as model materials for understanding the effect of surface chemistry in electrochemical properties and supercapacitor performance. However, the effect of nitrogen doping on the electrochemical performance of highly microporous N-doped ZTC in aqueous electrolyte and in supercapacitors application, has not been assessed before in the literature.

In this work, we show the differences on the performance of $\mathrm{N}$-doped and non-doped zeolite templated carbons through physicochemical and electrochemical characterization. The effect of nitrogen functional groups on the electrochemical behavior of these carbons in two different electrolytes ( $1 \mathrm{M} \mathrm{H}_{2} \mathrm{SO}_{4}$ and $\left.0.5 \mathrm{M} \mathrm{KOH}\right)$ is thoroughly analyzed by different techniques. Also, their performance as electrodes for supercapacitors is studied and related to the functional groups formed on each zeolite template carbon.

\section{EXPERIMENTAL}

\subsection{Zeolite templated carbons}

Zeolite templated carbons were synthesized by chemical vapor deposition of different precursors and using zeolite $\mathrm{Y}$ as a template (Na-form, $\mathrm{SiO}_{2} / \mathrm{Al}_{2} \mathrm{O}_{3}=5.6$, obtained from Tosoh Co. Ltd.) by following the method reported elsewhere $[6,28,29]$. As a result, two different ZTCs were obtained: non-doped zeolite templated carbon (ZTC) and N-doped zeolite templated carbon (N-ZTC) [23].

\subsection{Physicochemical characterization}

The structure order of the samples was analyzed by X-ray diffraction (XRD) recorded on Shimadzu XRD-6100 instrument with $\mathrm{Cu}-\mathrm{K} \alpha$ radiation. The porous texture was characterized by $\mathrm{N}_{2}$ physisorption technique carried out at $-196{ }^{\circ} \mathrm{C}$, by using a volumetric sorption analyzer (BEL Japan, BELSORP-max). The apparent specific surface area was calculated by the Brunauer-Emmett-Teller method $\left(\mathrm{S}_{\mathrm{BET}}\right)$ using the $\mathrm{N}_{2}$ adsorption data in the relative pressure $\left(\mathrm{P} / \mathrm{P}_{0}\right)$ range of $0.01-0.05$. The total micropore volume was calculated from the Dubinin-Radushkevich equation $\left(\mathrm{V}_{\mathrm{DR}}{ }^{\mathrm{N} 2}\right)$. 
The surface chemistry of the samples was analyzed by X Ray Photoelectron Spectroscopy (XPS) and Temperature Programmed Desorption (TPD). XPS analyses were performed by using a VGMicrotech Multilab 3000 spectrometer with an Al anode. N1s spectra were deconvoluted by using Gaussian functions with $20 \%$ of Lorentzian component. A Shirley line was used as background and the FWHM of the peaks was kept between 1.4 and $1.7 \mathrm{eV}$. TPD experiments were carried out by using a TGA-DSC instrument (TA Instruments, SDT Q600 Simultaneous) coupled to a mass spectrometer (Thermostar, Balzers, BSC 200), by heating the samples $(\sim 10 \mathrm{mg})$ up to $950{ }^{\circ} \mathrm{C}$ (heating rate: $20^{\circ} \mathrm{C} / \mathrm{min}$ ) under helium atmosphere (flow rate: $100 \mathrm{~mL} / \mathrm{min}$ ).

\subsection{Electrochemical characterization}

\subsubsection{Three electrode cell configuration}

Carbon electrodes for electrochemical characterization were prepared by mixing the carbon material with acetylene black and polytetrafluoroethylene (PTFE) as binder in a ratio of 90:5:5 (w/w). The total weight of the electrode was $\sim 9 \mathrm{mg}$ (dry basis). For shaping the electrodes, a sample sheet was cut into a circular shape with an area of $1.2 \mathrm{~cm}^{2}$ and pressed for $5 \mathrm{~min}$ at 2 tons to guarantee a homogeneous thickness. After that, the electrode was attached to a gold current collector by means of conducting adhesive (colloidal graphite suspension, Hitasol GA-715, Hitachi Chemical Co., Ltd.). The electrodes were soaked for 2 days into the electrolyte $\left(1 \mathrm{M} \mathrm{H}_{2} \mathrm{SO}_{4}\right.$ or $\left.0.5 \mathrm{M} \mathrm{KOH}\right)$ previously to electrochemical measurements.

The electrochemical characterization of the electrodes was performed by cyclic voltammetry in a Biologic VSP multichannel potentiostat and using a T-type Swagelock cell in a three-electrode configuration. $1 \mathrm{M} \mathrm{H}_{2} \mathrm{SO}_{4}$ was used as aqueous electrolyte. A capacitive electrode with more than twice the capacitance (electrode weight per gravimetric capacitance) of the working electrode was prepared from a commercial activated carbon and used as counter electrode. Both electrodes were tightly pressed against each other and separated by a nylon membrane (Teknokroma membrane filters, pore size: $450 \mathrm{~nm}) . \mathrm{Ag} / \mathrm{AgCl} / \mathrm{KCl}(3 \mathrm{M})$ was used as reference electrode in all cases. The electrochemical characterization of all samples was studied by cyclic voltammetry (CV) at sweep rate of $2 \mathrm{mV} / \mathrm{s}$. CV capacitance was calculated from the area of the voltammogram. The results are expressed in F/g, taking into account the weight of the active material of the working electrode.

\subsubsection{Two electrode cell configuration}

Symmetric capacitors (in mass) were assembled for both carbon materials. The electrodes were prepared by using the method described above (section 2.3.1) with a weight of $\sim 1.3 \mathrm{mg}$ (active 
phase) per electrode and a geometrical area of $0.196 \mathrm{~cm}^{2}$ (thickness: $0.2 \mathrm{~mm}$ ). The electrodes were attached to a stainless-steel collector following the previously mentioned protocol and using the same separator as described in section 2.3.1. These devices were characterized by $\mathrm{CV}$ at different scan rates, galvanostatic charge-discharge (GCD) cycles at current densities from 1 to $20 \mathrm{~A} / \mathrm{g}$ and Electrochemical Impedance Spectroscopy (EIS) in $1 \mathrm{M} \mathrm{H}_{2} \mathrm{SO}_{4}$ solution. Impedance spectra were measured at $0.05 \mathrm{~V}$ in the frequency range of $10 \mathrm{mHz}$ to $100 \mathrm{kHz}$ with an amplitude voltage of 10 $\mathrm{mV}$. The measurements were repeated in freshly prepared cells for their verification. Autolab PGSTAT302 potentiostat was employed for EIS and CV measurements and Arbin SCTS potentiostat for galvanostatic charge-discharge cycles. A durability test was performed by 50000 GCD cycles at a current density of $5 \mathrm{~A} / \mathrm{g}$ and a voltage of $1.2 \mathrm{~V}$. Current density and specific capacitance is defined based on the total active weight of the carbon material in the cell (two electrodes). The energy density and power density were calculated as described elsewhere [17].

\section{Results and discussion}

\subsection{Physicochemical characterization}

Table 1 summarizes the chemical properties of the samples. Although both samples are obtained by following the same procedure and the replica of the zeolite is successfully obtained providing an ordered microporous structure (see XRD patterns, Figure $\mathrm{S} 1$, and $\mathrm{N}_{2}$ adsorption-desorption isotherms, Figure S2), N-ZTC shows ca. $70 \%$ of the apparent surface area and micropore volume of ZTC.

The surface chemistry of both materials was characterized by XPS and TPD. The amount of nitrogen and oxygen detected using XPS and the evolved $\mathrm{CO}$ and $\mathrm{CO}_{2}$ quantities during TPD are compiled in Table 1. N-ZTC shows nitrogen content of 3.7 at. \% detected by XPS. According to the N1s XPS spectrum (Figure 1.b), this nitrogen is attached to the surface as the following functional groups: quaternary nitrogen $(401.2 \pm 0.2)$, pyrrole/pyridone $(400.5 \pm 0.2)$ and pyridines $(398.5 \pm 0.2)$. The percentage of each kind of functional group is $63 \%, 17 \%$ and $20 \%$, respectively. Hence, quaternary nitrogen is the most abundant nitrogen functionalities.

TPD experiments are used to study in detail the oxygen functionalities that exist on the surface of both carbons [30-33]. Both materials have a large amount of oxygen functional groups, ca. 3.5 $\mathrm{mmol} / \mathrm{g}$ or $5.5 \% \mathrm{wt}$ of oxygen. More specifically, $\mathrm{N}$-ZTC presents a larger amount of $\mathrm{CO}_{2}$-evolving groups in the $200-400{ }^{\circ} \mathrm{C}$ temperature range (Figure S3), which points out the presence of more carboxylic moieties in the nitrogen-doped sample. Interestingly, both samples show a similar amount of CO-evolving groups, but the TPD profile for the evolution of $\mathrm{CO}$, Figure 1a, evidences that the 
amount of phenolic and ether groups (related to the $\mathrm{CO}$ evolution at $600-700{ }^{\circ} \mathrm{C}$ ) is predominant in ZTC, whereas evolution of CO at higher temperatures is greater in N-ZTC. Since the CO evolution is still high at the end of the maximum temperature used in the TPD run, it can be considered that the total amount of oxygen functionalities is larger in this sample. Hence, surface oxygen groups of high thermal stability (such as carbonyls and/or even pyrenes) are found on its surface. In accordance to these findings, the surface oxygen functionalities present in ZTC has been previously studied by TPD and Fourier transform infrared spectroscopy and they have been assigned as: acid anhydride (15\%), ether (66\%), hydroxyl (15\%), and carbonyl (4\%), whereas N-ZTC presents a lower amount of ethers and hydroxyl functional groups along with a larger amount of carbonyls [7, 34].

Table 1. Textural properties and surface chemical composition (XPS and TPD) obtained for zeolite templated carbons.

\begin{tabular}{|c|c|c|c|c|c|c|}
\hline Sample & $\begin{array}{c}\text { S BET } \\
\left(\mathrm{m}^{2} / \mathrm{g}\right)\end{array}$ & $\begin{array}{c}V_{D R} N_{2} \\
\left(\mathrm{~cm}^{3} / g\right)\end{array}$ & $\begin{array}{c}\text { NXPS } \\
\text { (at.\%) }\end{array}$ & $\begin{array}{l}\mathrm{CO}_{2} \mathrm{TPD} \\
(\mu \mathrm{mol} / \mathrm{g})\end{array}$ & $\begin{array}{c}\text { CO TPD } \\
(\mu \mathrm{mol} / \mathrm{g})\end{array}$ & $\begin{array}{c}\text { O TPD }_{\text {TP }} \\
(\mu \mathrm{mol} / \mathrm{g})\end{array}$ \\
\hline ZTC & 3600 & 1.55 & - & 470 & 2330 & 3280 \\
\hline N-ZTC & 2760 & 1.05 & 3.7 & 510 & 2730 & 3750 \\
\hline
\end{tabular}
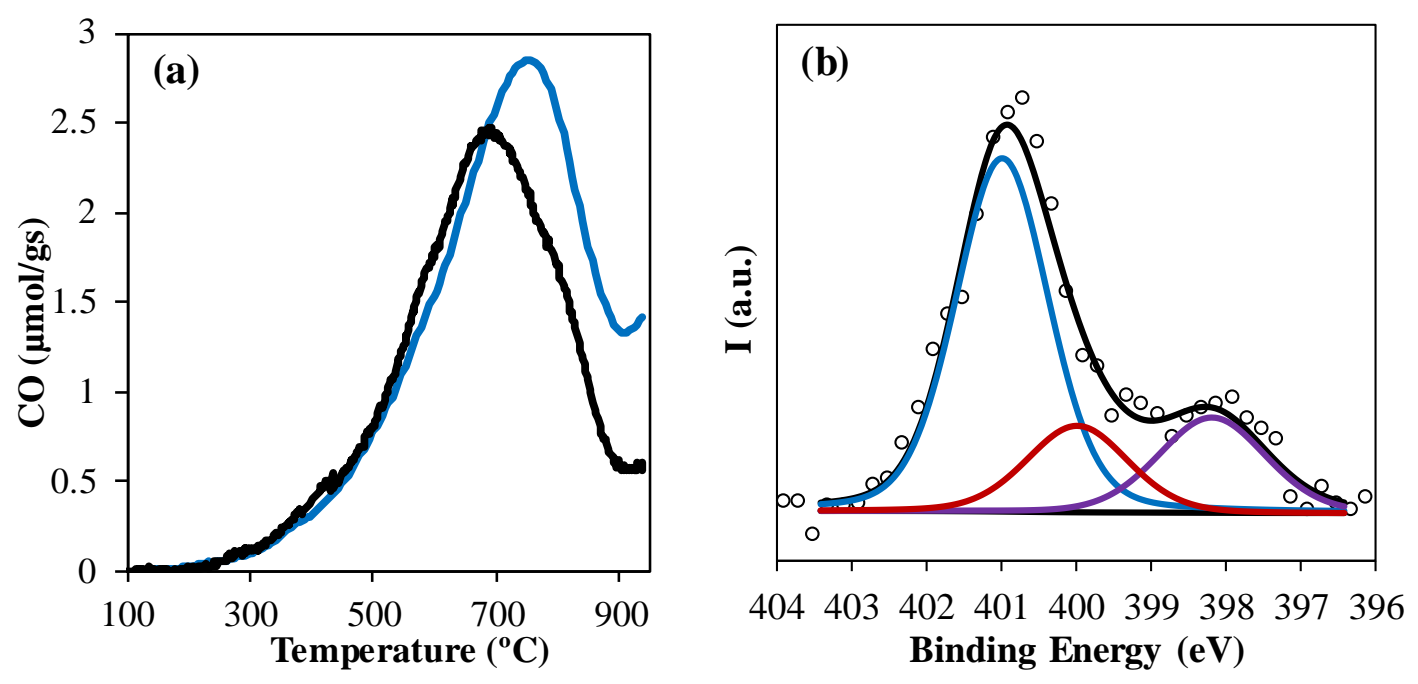

Figure 1. (a) Comparison between CO TPD profiles of ZTC (black line) and N-ZTC (blue line) samples. (b) N1s XPS deconvolution of N-ZTC sample. 


\subsection{Electrochemical characterization.}

\subsubsection{Electrochemical characterization before electro-oxidation}

The carbon materials were characterized in a three-electrode cell in acid and alkaline media in order to assess their electrochemical behavior. It should be noted that ZTC can be easily oxidized at low potentials in $1 \mathrm{M} \mathrm{H}_{2} \mathrm{SO}_{4}$, producing a very large amount of quinone functional groups that are responsible of a large boost in pseudocapacitance [6,35]. In order to avoid such feature, Figure $2 \mathrm{a}$ shows the CVs obtained for ZTC and N-ZTC in a potential range where no important oxidation occurs and the performance of both pristine carbon materials can be analyzed. The CVs obtained for both carbons have a broad oxidation peak during the positive sweep that reaches a maximum at 0.26 $\mathrm{V}$ for $\mathrm{N}-\mathrm{ZTC}$ and at $0.32 \mathrm{~V}$ for ZTC. At the negative sweep, the corresponding reduction peaks at potentials close to 0.16 and $0.23 \mathrm{~V}$ are observed respectively for N-ZTC and ZTC. The redox processes observed in the ZTC electrode are undoubtedly related to electroactive functional groups that, according to previous studies, correspond with the pseudocapacitive contribution of the quinone/hydroquinone redox pair [6]. However, N-ZTC shows larger pseudocapacitance in a wider potential range, delivering a capacitance $38 \mathrm{~F} / \mathrm{g}$ higher than that of ZTC (see $\mathrm{C}_{\mathrm{g}}$ values on Table 2). Since gravimetric capacitance is to some extent proportional to the surface area of porous carbon materials [36,37], this result must be a consequence of the presence of a higher amount of electroactive surface oxygen functionalities contained in this material together with nitrogen groups on N-ZTC.
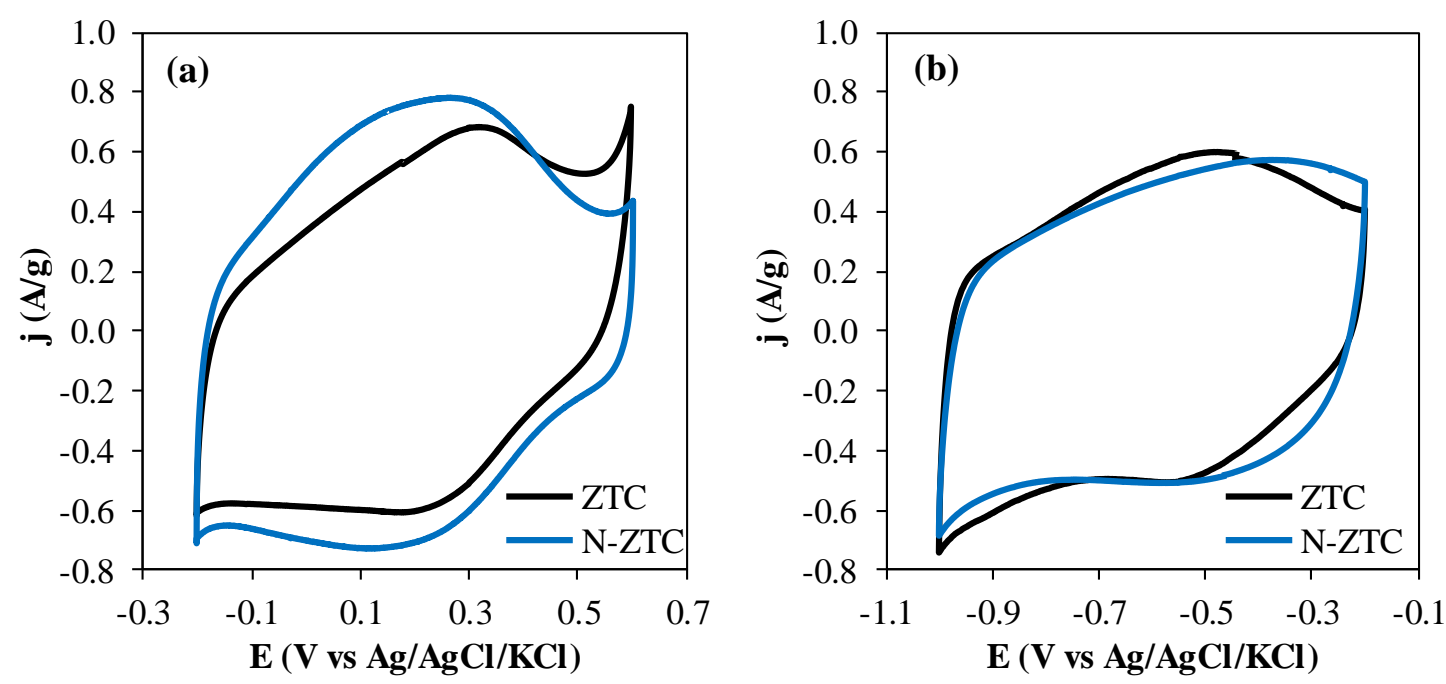
Figure 2. $3^{\text {rd }}$ cyclic voltammograms for ZTC and N-ZTC electrodes in (a) $1 \mathrm{M} \mathrm{H}_{2} \mathrm{SO}_{4}$ and (b) $0.5 \mathrm{M}$ $\mathrm{KOH} . \mathrm{v}=2 \mathrm{mV} / \mathrm{s}$.

Table 2. Gravimetric and normalized capacitances and equivalent series resistance of the samples.

\begin{tabular}{|c|c|c|c|c|c|c|c|}
\hline Sample & $\begin{array}{c}\mathrm{C}_{\mathrm{g}}\left[\mathrm{H}_{2} \mathrm{SO}_{4}\right] \\
(\mathrm{F} / \mathrm{g})\end{array}$ & $\begin{array}{c}\mathrm{C}_{\mathrm{g}} \\
{[\mathrm{KOH}]} \\
(\mathrm{F} / \mathrm{g})\end{array}$ & $\begin{array}{c}\mathrm{C}_{\mathrm{g}} / \mathrm{S}_{\text {BET }} \\
{\left[\mathrm{H}_{2} \mathrm{SO}_{4}\right]} \\
\left(\mu \mathrm{F} / \mathrm{cm}^{2}\right)\end{array}$ & $\begin{array}{c}\mathrm{C}_{\mathrm{g}} / \mathrm{S}_{\mathrm{BET}} \\
{[\mathrm{KOH}]} \\
\left(\mu \mathrm{F} / \mathrm{cm}^{2}\right)\end{array}$ & $\begin{array}{c}\mathbf{C g}^{\text {ox }} \\
{\left[\mathrm{H}_{2} \mathrm{SO}_{4}\right]} \\
(\mathrm{F} / \mathrm{g})\end{array}$ & $\begin{array}{c}\mathrm{Cg}^{\mathrm{ox}} \\
{[\mathrm{KOH}]} \\
(\mathrm{F} / \mathrm{g})\end{array}$ & $\begin{array}{c}\mathrm{ESR} \\
{\left[\mathrm{H}_{2} \mathrm{SO}_{4}\right]} \\
(\Omega)\end{array}$ \\
\hline ZTC & 235 & 232 & 6.0 & 5.9 & 445 & 246 & 1.8 \\
\hline N-ZTC & 273 & 224 & 9.9 & 8.1 & 384 & 230 & 0.3 \\
\hline
\end{tabular}

In order to achieve a better understanding of the origin of the higher capacitance of N-ZTC, an analogous CV was recorded in basic medium (Figure 2.b) for both materials. In this case, the intensity of the redox peaks previously seen in acid medium is notoriously decreased. Hence, the pseudocapacitance observed in acid medium is a consequence of functionalities that are not electroactive in basic medium. In this sense, it has been reported that pyridine groups can contribute to largest capacitance in basic media than in acid media via 1 electron process that is impeded in acid electrolyte due to its protonation in this medium $[8,38]$. This is the opposite trend observed for $\mathrm{N}$ ZTC. However, it must be considered that, since the presence of pyridinic nitrogen in N-ZTC is low, it should not be expected that they have a high contribution to capacitance in alkaline electrolyte.

Thus, the highest pseudocapacitive response of pristine N-ZTC in acid electrolyte should be a consequence of its oxygen functionalities. As seen in section 3.1, N-ZTC has larger amount of carbonyl functional groups along with other CO-evolving oxygen functionalities of higher thermal stability (Figure 1a). These functional groups could take part in redox reactions involving protons [11], explaining why the pristine N-ZTC shows both a higher capacitance than ZTC in acid media and a capacitance decrease in alkaline electrolyte.

Also, it should be noted that the surface capacitance $\left(\mathrm{C}_{\mathrm{g}} / \mathrm{S}_{\mathrm{BET}}\right.$ in Table 2$)$ is larger for N-ZTC in both media. Since the pseudocapacitance contribution is mostly neglected in alkaline media, the difference in surface capacitance in $0.5 \mathrm{M} \mathrm{KOH}$ can be related to an improvement of wettability as consequence of the different surface chemistry. This improvement can be a consequence of oxygen or nitrogen functional groups. It is well-known that CO-evolving groups increase the wettability of carbon materials [11]. Also, the contribution of N-Q to the wettability of carbons was previously 
reported $[8,39,40]$. Since both moieties exist on larger amounts on N-ZTC, the improvement in surface capacitance can be related to an enhanced wettability of this carbon material. Moreover, the distribution of the functional groups is expected to be different for N-ZTC and ZTC, with N-Q being found inside the pore network, increasing the wettability of the whole surface of N-ZTC, whereas the CO-evolving groups of ZTC are expected to be preferentially located at edge sites at the entrance of pores, delivering a lower wetting of the inner porosity.

\subsubsection{Electrochemical characterization after electro-oxidation}

This section reports the performance of zeolite templated carbons after exposing them to electrooxidation conditions in acid and alkaline media. Both carbons were electro-oxidized in $1 \mathrm{M} \mathrm{H}_{2} \mathrm{SO}_{4}$ by performing three $\mathrm{CVs}$ between -0.2 and $1.0 \mathrm{~V}$ at $2 \mathrm{mV} / \mathrm{s}$. Figure 3 shows these $\mathrm{CVs}$ for both carbon materials. On the first anodic sweep, both electrodes show a large irreversible oxidation current when the potential is shifted to values more positive than $0.6 \mathrm{~V}$. It is well stablished that this oxidation treatment produces electro-active oxygen functional groups in zeolite templated carbons [6,28, 41], and also some degradation of the structure and electro-gasification [32]. The oxidation current is larger for the ZTC electrode (see the higher intensity of the black CV at $1.0 \mathrm{~V}$ in Figure 3.a compared to that of Figure 3.b), evidencing that more intensive oxidation occurs in this sample. The net increase of the currents of the reversible peaks at ca. $0.3 \mathrm{~V}$, which are related to electroactivity of the quinone/hydroquinone functionalities, observed after the electro-oxidation treatments corroborates this phenomenon (see the differences between red -initial- and purple -final- CVs in Figure 3a).

N-ZTC also shows the same features (Figure 3b). However, the observed increase of these redox processes is lower. Consequently, the capacitance recorded for ZTC after electro-oxidation $\left(\mathrm{C}^{\mathrm{ox}}\right.$, Table 2) is larger than that found for N-ZTC. It is interesting to note that the voltammograms after the oxidation show different redox processes for both carbon materials. Then, in ZTC an increase in the voltammetric charge is observed in a wide potential window, from -0.1 up to $0.6 \mathrm{~V}$, while in the case of N-ZTC, the increase in voltammetric charge is only observed from $0.2 \mathrm{~V}$ to $0.7 \mathrm{~V}$. More significant differences upon the electro-oxidation between these materials are highlighted by overlapping their CVs (Figure 4). Although both CVs show similar features, ZTC does show a larger pseudocapacitance from 0.05 to $0.4 \mathrm{~V}$. Since the structure of both materials is essentially identical [23] (see XRD patterns, Figure S2), their different performance under electro-oxidative conditions should be related to their different surface chemistry. The electro-oxidized electrodes were analyzed by TPD and it was found that approximately the same amount of oxygen functionalities (6690 
$\mu \mathrm{mol} / \mathrm{g}$ for ZTC and $6740 \mu \mathrm{mol} / \mathrm{g}$ for for N-ZTC) are generated in the surface of both materials after the treatment. Itoi et al [6] proposed that the incorporation of oxygen groups on ZTC upon the electro-oxidation happens because of the large amount of reactive edge sites that exist on these carbons. Since N-ZTC incorporates the same amount of oxygen functional groups, it can be inferred that larger irreversible oxidation currents experimented by ZTC may be connected to gasification reactions, where certain oxygen functionalities may evolve as $\mathrm{CO}$ and $\mathrm{CO}_{2}$ (and therefore, their presence is not detected by TPD over the oxidized electrode) during the CV sweep. Moreover, the electroactivity of certain functional groups showed by electro-oxidized ZTC seem to be hindered or even impeded in N-ZTC, since both carbons have developed a similar amount and type of oxygen functionalities.
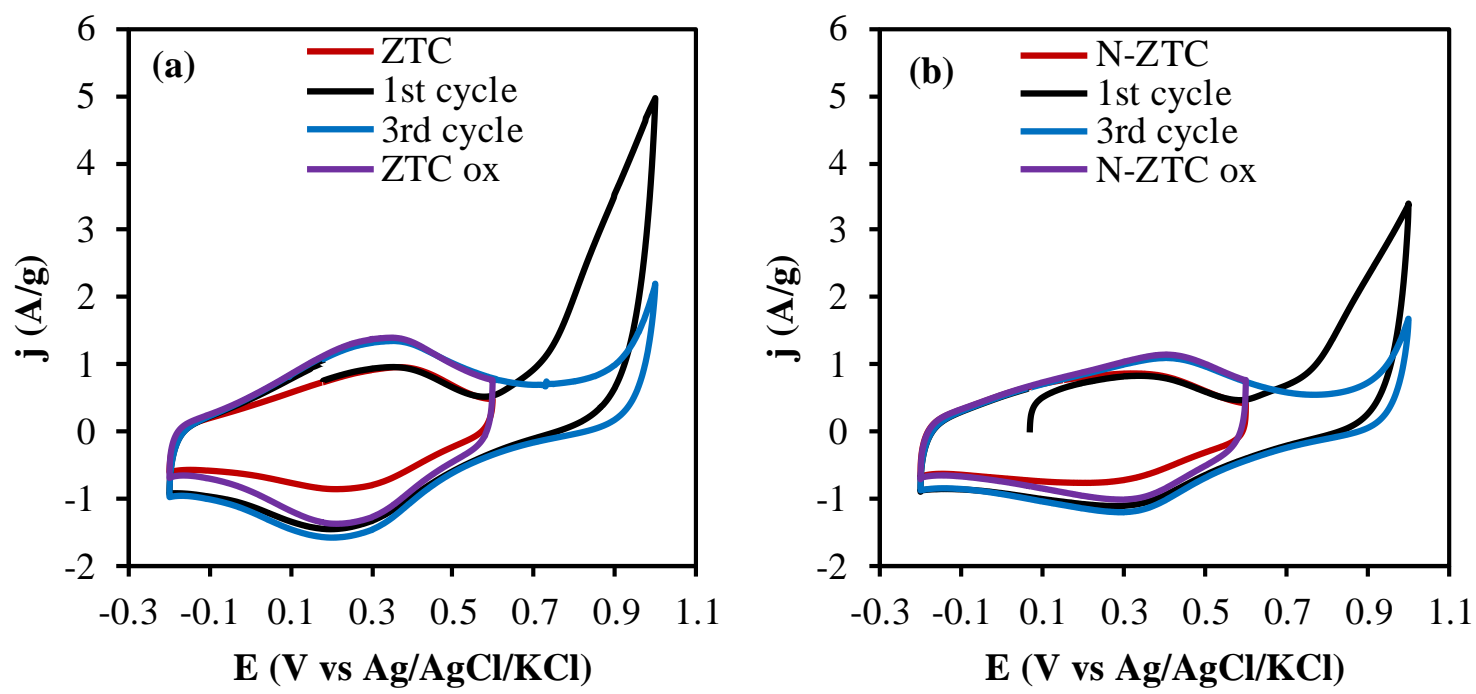

Figure 3. Cyclic voltammograms for the electro-oxidation of ZTC (a) and N-ZTC (b). $v=2 \mathrm{mV} / \mathrm{s}$. $1 \mathrm{M} \mathrm{H}_{2} \mathrm{SO}_{4}$. 


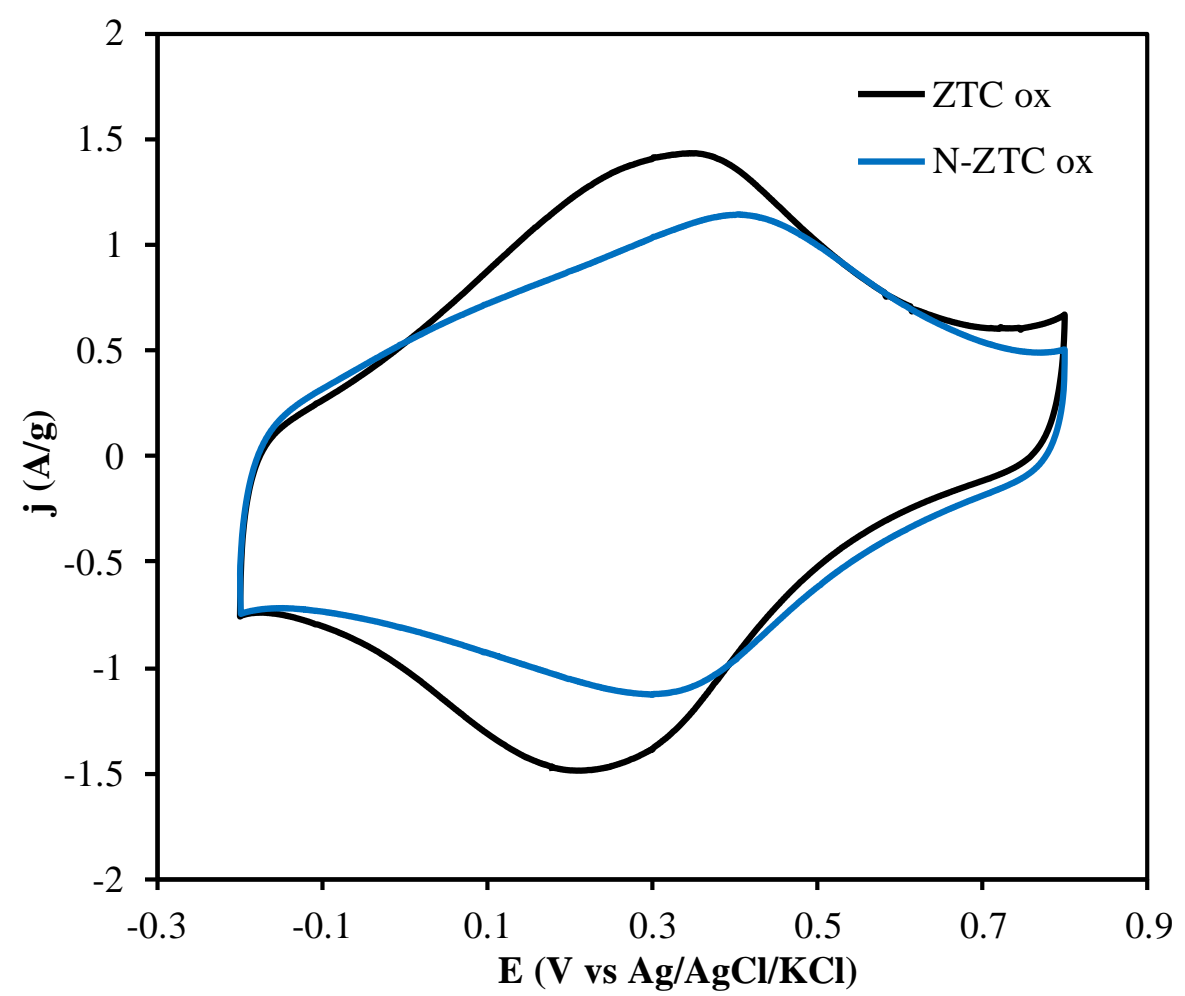

Figure 4. $3^{\text {rd }}$ cyclic voltammograms for ZTC and N-ZTC after electro-oxidation. $\mathrm{v}=2 \mathrm{mV} / \mathrm{s} .1 \mathrm{M}$ $\mathrm{H}_{2} \mathrm{SO}_{4}$.

EIS measurements were carried out for the electro-oxidized samples in order to deepen into the changes produced by the electrochemical oxidation. This technique allows one to distinguish the different resistance contributions affecting the electrodes (diffusive problems, electrode-electrolyte interface, etc.) [42]. Figure 5 shows the Nyquist plots obtained in $1 \mathrm{M} \mathrm{H}_{2} \mathrm{SO}_{4}$ for both samples after the electro-oxidation in the acid electrolyte. The main differences between both profiles are observed at low frequencies, where an almost vertical line, related to capacitive behavior, is observed. The onset frequency for reaching capacitive behavior is higher in the case of N-ZTC. The equivalent series resistance (ESR) plus the equivalent distributed resistance (EDR) of the cells was calculated from the $\mathrm{x}$-intercept of this line. The values obtained for both electrodes are summarized in Table 2. It can be observed that ZTC electrode shows a higher resistance than N-ZTC and a remarkably Warburg region, related to diffusion processes through the pore network [43]. In this work, the electrodes and the electrochemical cells were prepared by following the same procedure and, consequently, the differences observed are related to the electrode materials. As discussed before (section 3.1), the zeolite templated carbons in this study initially have a similar ordered structure and the same pore size of $1.2 \mathrm{~nm}$. The main differences of both carbons are their surface chemistry and the larger apparent surface area of non-doped ZTC. In this sense, N-ZTC has an important content in 
$\mathrm{N}$-functionalities that improve both electrical conductivity and wettability [20,44-46], which can explain a decrease in the electrolyte diffusion resistance.

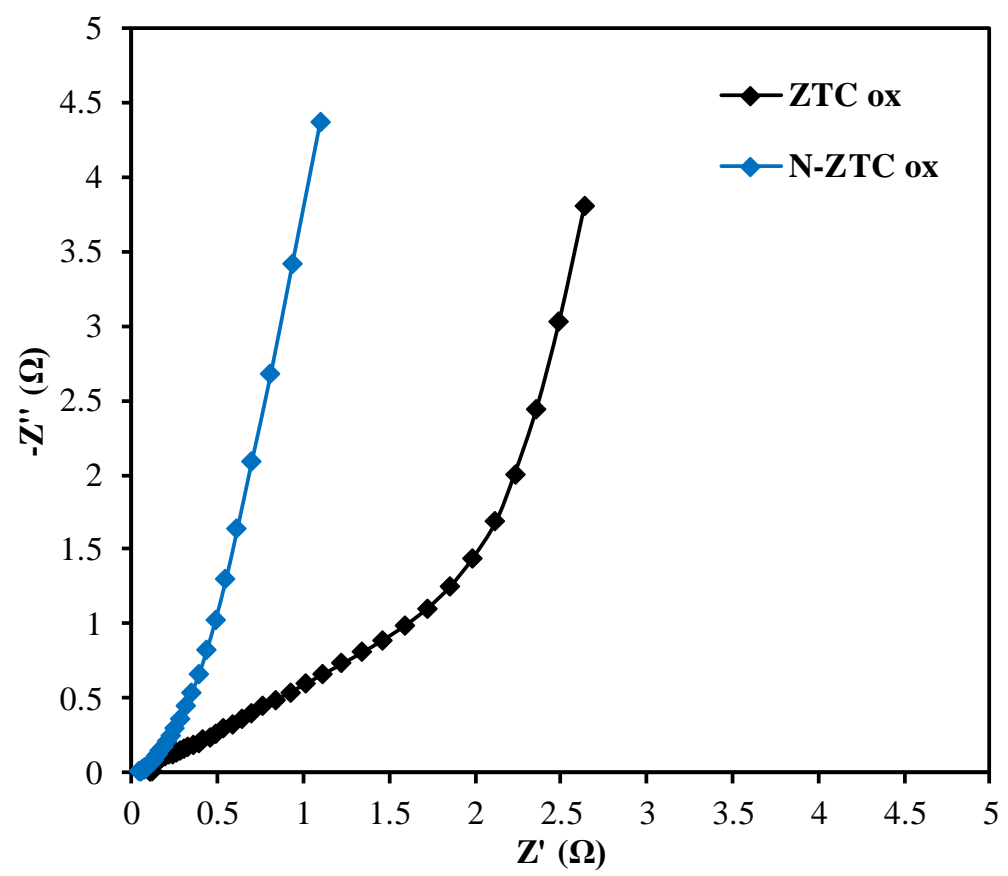

Figure 5. Electrochemical impedance spectra for ZTC and N-ZTC electrodes after electro-oxidation in $1 \mathrm{M} \mathrm{H}_{2} \mathrm{SO}_{4} . \mathrm{E}=0.3 \mathrm{~V}$ vs $\mathrm{Ag} / \mathrm{AgCl}$.

The differences in electrochemical stability when exposed to high oxidation potentials were further studied in alkaline electrolyte. Figure 6 shows the cyclic voltammograms obtained for N-ZTC and ZTC in basic medium under the oxidative conditions. Again, the current density increases when the potential is shifted to more positive values and an irreversible faradaic current related to oxidation processes is observed (black curve, Figure 6.a and b). As happened in acid medium, the current density obtained for ZTC is higher than for N-ZTC electrode, pointing out the higher electrochemical stability of the $\mathrm{N}$-doped carbon.

Furthermore, the CV obtained for N-ZTC remains practically invariable after positive polarization, while, in case of ZTC, there is a small increase of capacitance (Table 2). This increase of capacitance can be explained by different phenomena: (i) increase of wettability and (ii) appearance of pseudocapacitance. In general, carbon materials have an increase of wettability when oxygen functional groups (mainly CO-evolving groups) are attached to their surface [47]. Consequently, the improvement of capacitance of ZTC is mainly attributed to the generation of oxygen functionalities on this carbon after electro-oxidation. As expected, this effect is not observed in N-ZTC electrode owing to the contribution of N-Q functionalities to the wettability of this material. Regarding 
pseudocapacitance, quinones are reported to be electroactive at lower pHs. However, some small pseudocapacitive behavior is observed in alkaline electrolyte for both N-ZTC and ZTC electrodes, Figure 7. Previous research reported the evidence of pseudocapacitance in alkaline medium due to the contribution of oxygen functionalities in carbon cloths when the $\mathrm{pH}$ is above 11 due the previous activation in acid medium of pyrone derivatives and another unidentified species [48]. Thus, different $\mathrm{CO}-$ evolving functional groups could be responsible of the electroactivity showed in $\mathrm{KOH}$ electrolyte by both carbon materials, although this is an issue that needs further research. 

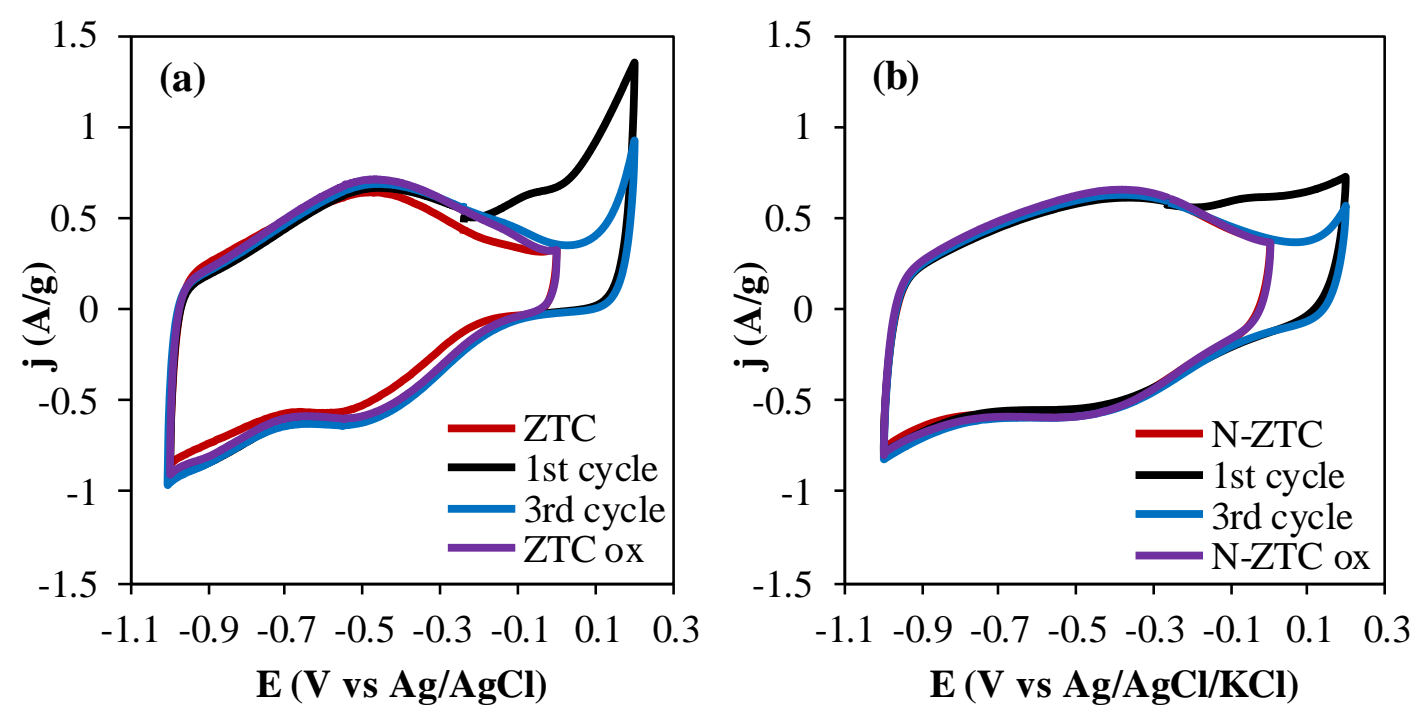

Figure 6. Cyclic voltammograms for the electro-oxidation of ZTC (a) and N-ZTC (b). $v=2 \mathrm{mV} / \mathrm{s}$. $0.5 \mathrm{M} \mathrm{KOH}$.

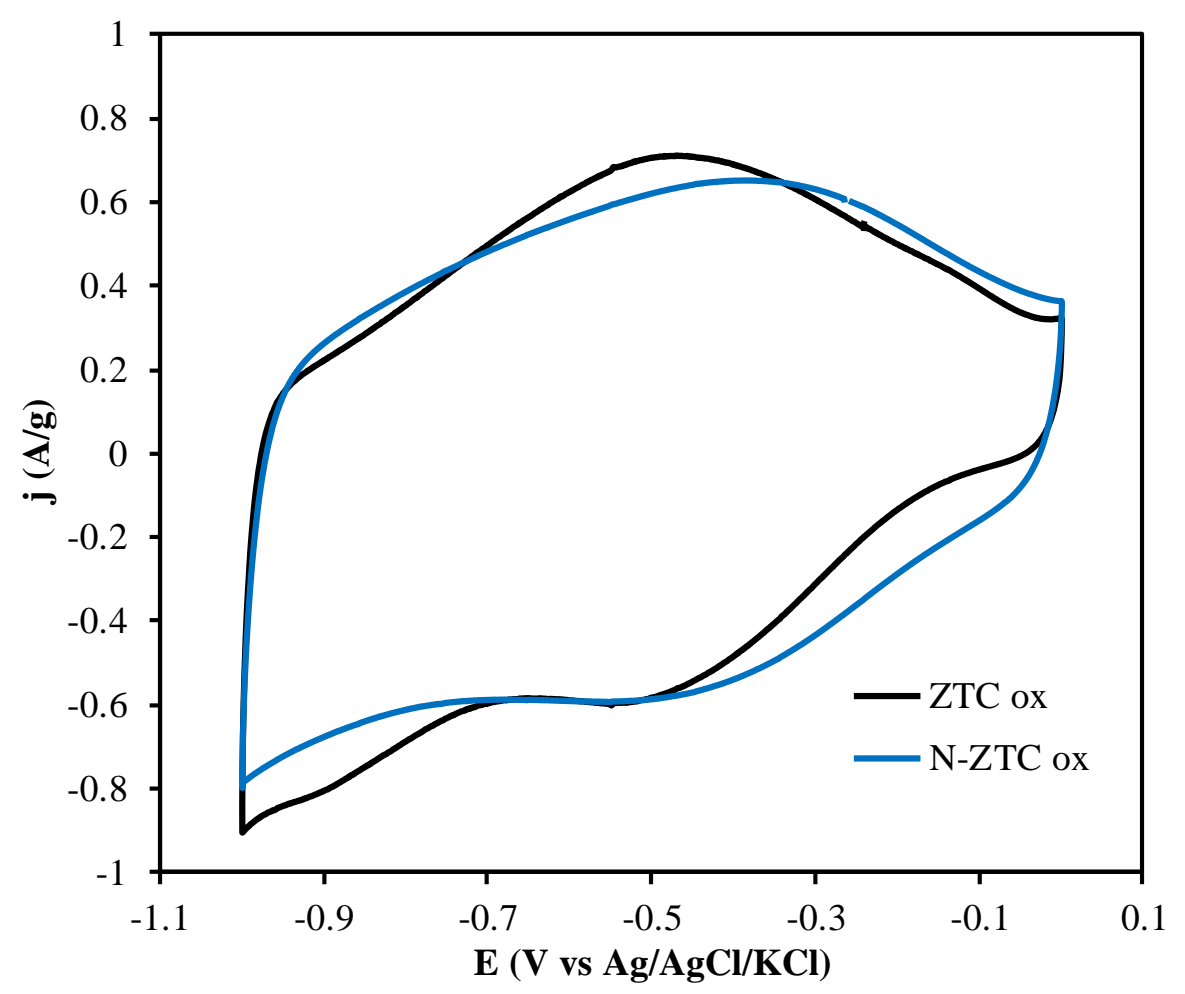

Figure 7. $3^{\text {rd }}$ cyclic voltammograms for ZTC (a) and N-ZTC (b) after electro-oxidation. $\mathrm{v}=2 \mathrm{mV} / \mathrm{s}$. $0.5 \mathrm{MKOH}$. 


\subsubsection{ZTC and N-ZTC supercapacitors using acid electrolyte}

The performance of the materials as electrodes for supercapacitors was assessed by using a symmetric configuration (in mass) in $1 \mathrm{M} \mathrm{H}_{2} \mathrm{SO}_{4}$. Prior to assembling the two-electrode cell of ZTC, both positive and negative electrodes were electro-oxidized (as described in section 3.2.2) in order to stabilize their response and avoid any problem related with the large irreversible current shown by ZTC during the first loading cycles [49]. Since the electro-oxidation current shown by N-ZTC in the $\mathrm{CV}$ studies was lower, the stability of the negative electrode is not affected (i.e. the negative electrode does not reach potentials where hydrogen evolution occurs) and, consequently, this pretreatment was not needed for N-ZTC electrodes.

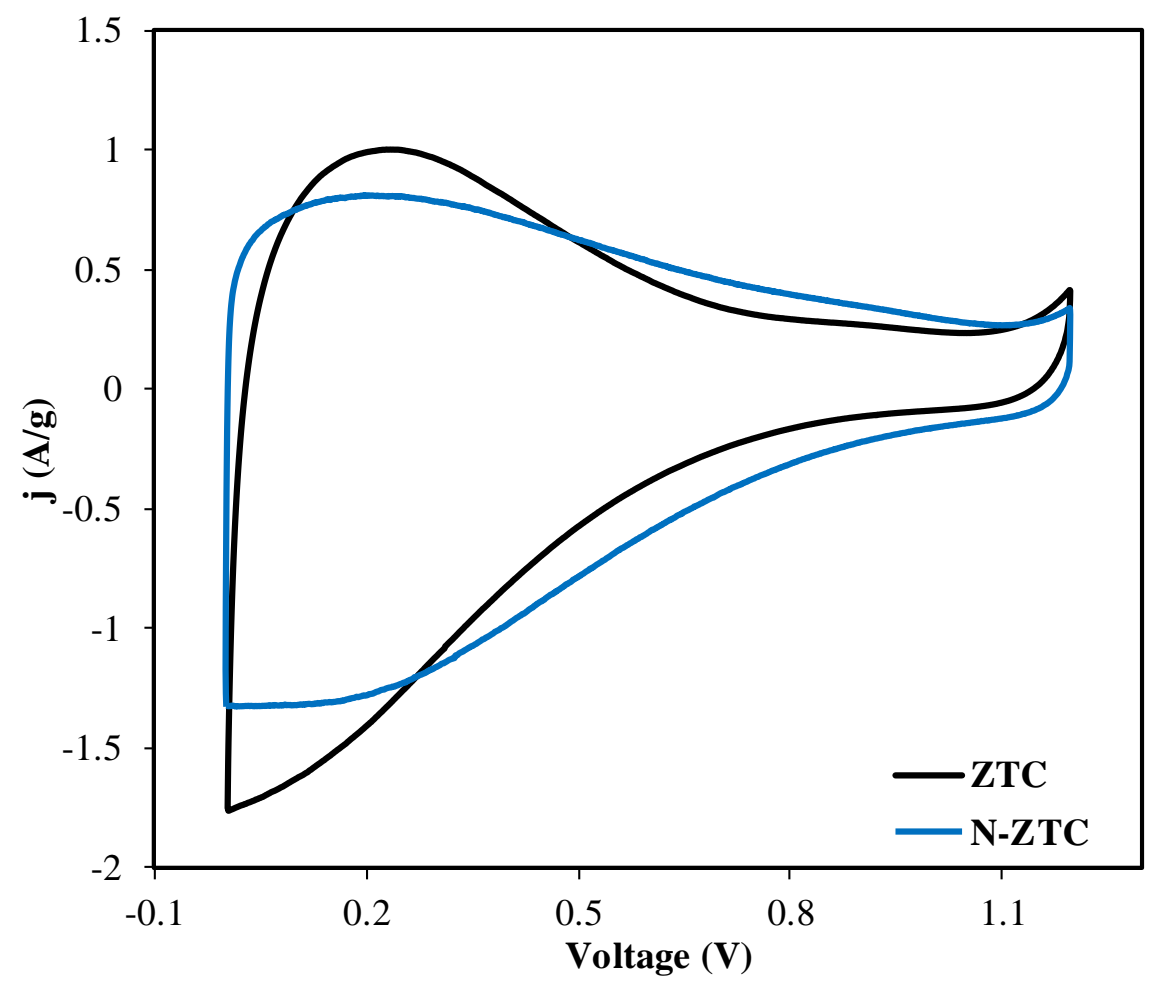

Figure 8. Steady state cyclic voltammograms for ZTC and N-ZTC based supercapacitors. $\mathrm{v}=10$ $\mathrm{mV} / \mathrm{s} . \mathrm{V}=1.2 \mathrm{~V} .1 \mathrm{M} \mathrm{H}_{2} \mathrm{SO}_{4}$.

Figure 8 shows cyclic voltammograms obtained for ZTC and N-ZTC based capacitors operating at $1.2 \mathrm{~V}$ and Table 3 compiles the gravimetric capacitance obtained from these CVs. The main contribution to the gravimetric capacitance is given at low voltage, since the electrodes in the capacitors are working around the open circuit potential (OCP, 0.34 and $0.41 \mathrm{~V}$ for ZTC and N-ZTC electrodes, respectively), where the pseudocapacitance contribution is maximized. As the voltage increases, the current decreases sharply, until reaching approximately a constant value when the voltage is beyond $0.7 \mathrm{~V}$. At these voltages, the potentials of positive and negative electrodes are 
expected to be shifted at least $0.35 \mathrm{~V}$ from the OCP (since the voltage of the cell results from the difference of potential between both electrodes). Thus, they have reached potentials where these materials mainly have the contribution of electrical double layer (Figures 3 and 4), and therefore a much lower capacitance is obtained from each electrode and, accordingly, in the supercapacitor.

In the case of N-ZTC supercapacitor, the charge is lower than for ZTC at voltages close to zero due to the lower pseudocapacitance contribution on this material (Figure 4), while the charge drop of the supercapacitor at medium and high voltages in N-ZTC is mitigated due to the higher charge of this material as the potential of the electrodes is shifted from OCP.

Table 3. Parameters obtained for ZTC and N-ZTC based capacitors in $1 \mathrm{M} \mathrm{H}_{2} \mathrm{SO}_{4}$.

\begin{tabular}{cccccccc}
\hline Supercapacitor & $\mathbf{C}$ CV & $\mathbf{C}_{\mathbf{G C D}}$ & $\mathbf{C}_{\mathbf{G C D}}$ & $\mathbf{R}$ & $\mathbf{E}$ & $\mathbf{P}_{\max }$ & $\mathbf{C}_{\mathbf{f}} / \mathbf{C}_{\mathbf{o}}$ \\
& $(\mathbf{F} / \mathbf{g})$ & {$[\mathbf{1 ~ A} / \mathbf{g}]$} & {$[\mathbf{2 0} \mathbf{A} / \mathbf{g}]$} & $(\mathbf{\Omega})$ & $(\mathbf{W h} / \mathbf{k g})$ & $(\mathbf{k W / k g})$ & \\
& & $(\mathbf{F} / \mathbf{g})$ & $(\mathbf{F} / \mathbf{g})$ & & & & \\
\hline ZTC & 60 & 61 & 19 & 3.50 & 5.9 & 23 & 88 \\
N-ZTC & 56 & 57 & 46 & 0.85 & 7.5 & 98 & 91 \\
\hline
\end{tabular}



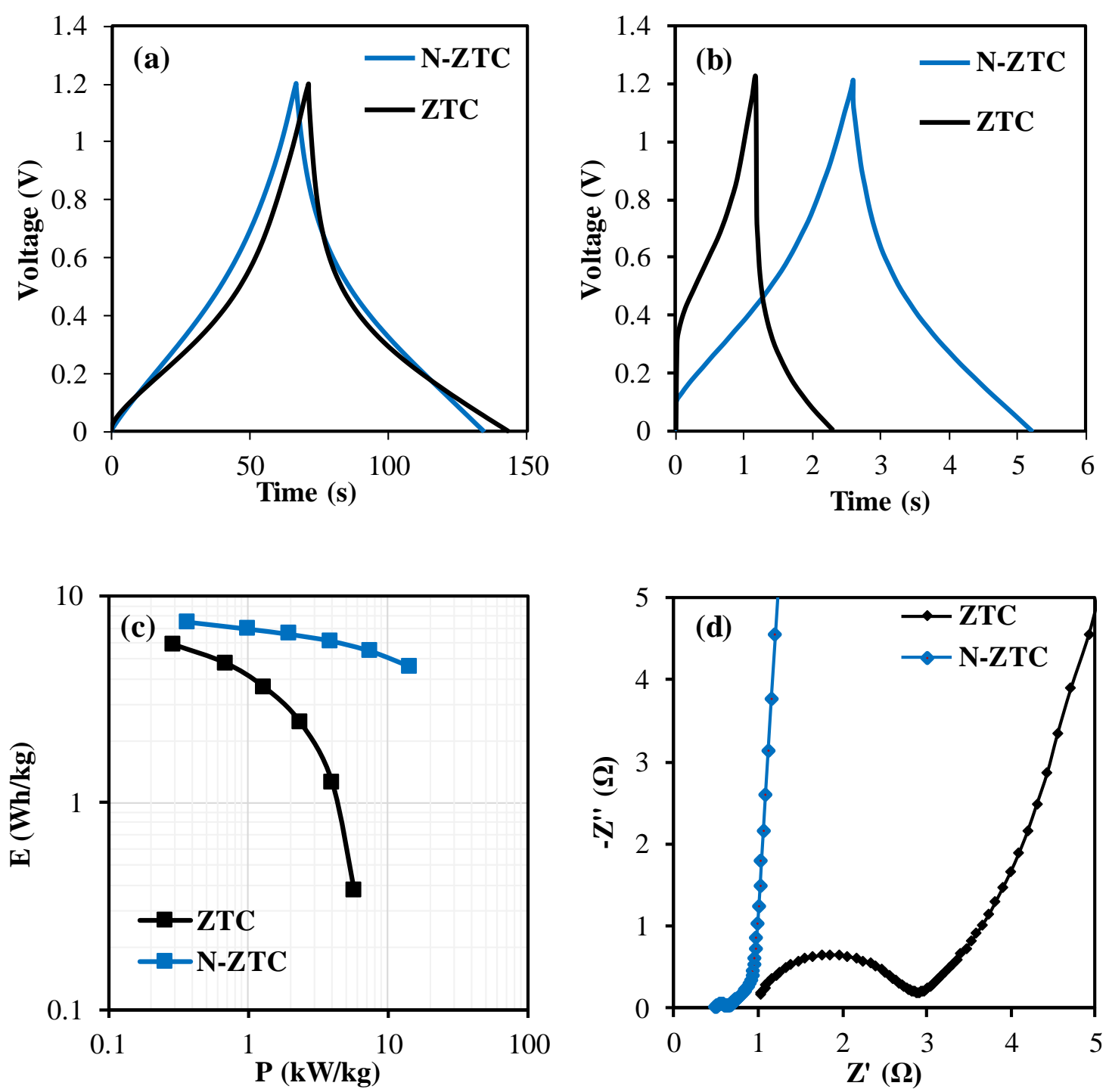

Figure 9. GCD cycles for ZTC and N-ZTC based supercapacitors at (a) $1 \mathrm{~A} / \mathrm{g}$ and (b) $20 \mathrm{~A} / \mathrm{g}$. V = 1.2 V. (c) Ragone plot obtained for ZTC and N-ZTC based supercapacitors at 1, 2.5, 5, 10 and 20 A/g. V = 1.2 V. (d) Electrochemical impedance spectra for ZTC and N-ZTC based supercapacitors. $\mathrm{V}=0.05 \mathrm{~V} .1 \mathrm{M} \mathrm{H}_{2} \mathrm{SO}_{4}$. 
Figure 9.a shows the galvanostatic charge-discharge (GCD) cycles obtained for ZTC and N-ZTC based capacitors at $1 \mathrm{~A} / \mathrm{g}$. The shape of the cycles differs from the ideal triangle characteristic of carbon materials with pure contribution of electrical double layer formation due to the strong influence of pseudocapacitive processes and other phenomena previously discussed (see section 3.2.1). Under these conditions, both cells evidence similar gravimetric capacitance (Table 3). However, the increase of current density up to $20 \mathrm{~A} / \mathrm{g}$ produces a decrease in capacitance more accused in the case of ZTC, due to a higher ohmic drop (Figure 9.b). This plays an important role on the retention of energy at high power density, as can be seen in the Ragone plot (Figure 9.c). Thus, when energy is considered, N-ZTC based supercapacitor shows only a small improvement compared to ZTC one at $1 \mathrm{~A} / \mathrm{g}$. However, the difference in energy becomes larger at high current density, since $\mathrm{N}-\mathrm{ZTC}$ based capacitor only experiences a decrease of $28 \%$ of the initial energy at $20 \mathrm{~A} / \mathrm{g}$, while in case of ZTC cell, a loss of $78 \%$ is observed. These differences on the energy retention at high power density are usually related to certain properties of the electrodes, such as electrical conductivity and ion mobility within the porosity. These properties can be better understood by EIS.

Figure 9.d shows the Nyquist plot obtained for ZTC and N-ZTC based capacitors at V $=0.05 \mathrm{~V}$ recorded after finishing the determination of the Ragone plot. The profiles shown in Figure 9.d are characteristic of carbon materials with a mainly capacitive behavior [43]. At high frequencies, a semicircle is observed in both supercapacitors. This semicircle is produced by the parallel combination of the bulk capacitance of the electrolyte and the electrical resistances to charge propagation in the electrode [50], and it includes contact resistance between particles. At medium frequencies, the Warburg region (evidenced by a $45^{\circ}$ line in the Nyquist plot) is observed, while at low frequencies, the shape of the curve becomes close to a vertical line, characteristic of the capacitive behavior [42]. Table 3 summarizes the cell resistance obtained for both capacitors. As happened in three electrode cell configuration (section 3.2.2.), N-ZTC capacitor shows much lower resistance. These cell resistances are governed by: (i) the diffusive problems of the ions through the porosity (Warburg region [43]) and (ii) the electrical resistances to charge propagation in the electrode (indicated by the diameter of the semicircle) [50]. Both the Warburg and the semicircle resistances are lower in N-ZTC, although the latter resistance is much lower. For understanding the difference in this resistance, it should be noted that both ZTC and N-ZTC supercapacitors have been assembled using the same electrolyte, the same electrode composition and by following the same procedure. Therefore, we proposed that the main differences between the cell resistances of these supercapacitors are mainly related to the inherent electrical conductivity of the electrode materials 
that decrease the contact resistance between particles. As discussed before (section 3.2.2), the main differences of both carbons are related to the presence of nitrogen heteroatoms in N-ZTC. The resistance value obtained for this capacitor is four times lower than that calculated for ZTC cell (Table 3), and it evidences the better electrical conductivity and charge propagation of N-ZTC electrodes. In accordance to this finding, the maximum power obtained for N-ZTC based capacitor (calculated from the resistance measured from the ohmic drop of GCD cycles, which is in good agreement to that obtained from the EIS analyses) is four times larger than the value obtained for ZTC cell. This value outperforms those found in the literature for other carbon electrodes in supercapacitors [5,51,52], such as activated carbons $\left(61.2 \mathrm{~kW} / \mathrm{kg}\right.$ in $\left.1 \mathrm{M} \mathrm{H}_{2} \mathrm{SO}_{4}\right)$ [17], activated carbon nanofibers $(20 \mathrm{~kW} / \mathrm{kg}$ in $6 \mathrm{M} \mathrm{KOH})$ [53], hierarchical porous carbons $(52.7 \mathrm{~kW} / \mathrm{kg}$ in $1 \mathrm{M}$ $\mathrm{H}_{2} \mathrm{SO}_{4}$ ) [54], carbon nanotubes (43.3 kW/kg in $1 \mathrm{M} \mathrm{Et}_{4} \mathrm{NBF}_{4} /$ propylene carbonate) [55] and other templated carbons $\left(28 \mathrm{~kW} / \mathrm{kg}\right.$ in $\left.1 \mathrm{M} \mathrm{H}_{2} \mathrm{SO}_{4}\right)$ [56]. This outstanding improvement of $\mathrm{N}-\mathrm{ZTC}$ based capacitor is probably a consequence of its connected nanopore structure, which facilitates the accessibility of the electrolyte [4], and the large quantity of N-Q functionalities and pyrrole on the surface of this material, that are able to increase both wettability and electrical conductivity $[18,20$, $44,45]$.

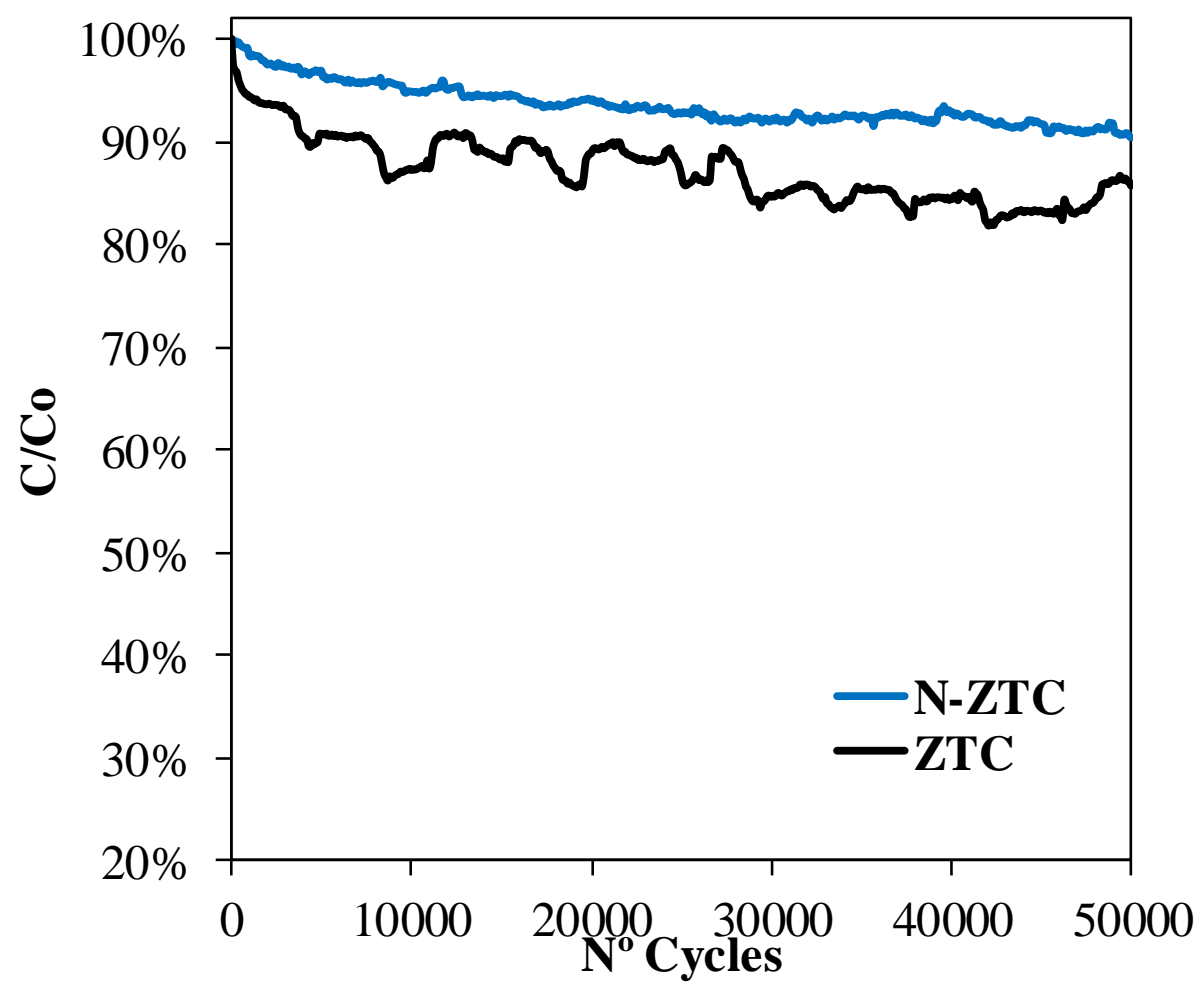

Figure 10. Durability test for ZTC and N-ZTC based supercapacitors at $1.2 \mathrm{~V} .5 \mathrm{~A} / \mathrm{g}$. 50000 cycles. 1 $\mathrm{M} \mathrm{H}_{2} \mathrm{SO}_{4}$ electrolyte. 
The presence of nitrogen functionalities can also modify the stability of porous carbon materials [19][22][17]. In order to explore this option, the durability of both cells was evaluated by 50000 GCD cycles at $1.2 \mathrm{~V}$. Figure 10 shows the evolution of the capacitance retention $\left(\mathrm{C} / \mathrm{C}_{\mathrm{o}}\right)$ during the test. Both capacitors evidence a similar capacitance retention after the durability test (Table 3), although it is somewhat higher in case of N-ZTC cell. However, it can be seen in Figure 10 that ZTC based capacitor shows larger changes of capacitance along cycles, evidencing a less stable performance. The improvement of stability in this capacitor is probably a consequence of the $\mathrm{N}$ functionalities formed on N-ZTC electrodes, since they are able to increase the stability by preventing the incorporation of detrimental oxygen functionalities [17].

\section{CONCLUSIONS}

In this work, the electrochemical behavior of non-doped and N-doped zeolite templated carbons were studied as electrodes for supercapacitors in different aqueous electrolytes. The materials were synthesized by CVD of different precursors and they evidenced a practically identical structure but different surface chemistry. The role of the different $\mathrm{N}$-functionalities in their electrochemical performance was elucidated by different techniques. The study carried out by cyclic voltammetry evidenced a higher resistance to electro-oxidation and degradation in case of N-ZTC in acid and alkaline media. Also, the different electroactivity in $1 \mathrm{M} \mathrm{H}_{2} \mathrm{SO}_{4}$ of the functional groups generated at positive potentials was demonstrated. The CV study suggested that N-ZTC has better wettability than ZTC as consequence of the large amount of N-Q functionalities at its surface. Also, it was shown that N-Q functionalities provide better conductivity to this carbon.

The effect of the different performance of these carbons as electrodes for supercapacitors was assessed in acid electrolyte using a two-electrode cell configuration. The results showed that both capacitors provide similar capacitance, but larger energy in case of N-ZTC. This result is the outcome of the large dependence of capacitance on potential in the highly oxidized ZTC. This behavior produces a large capacitance at low voltages, but a much smaller capacitance at high voltages, where most energy would be stored. In consequence, the more capacitive behavior of $\mathrm{N}$ ZTC renders an improved energy density.

More interestingly, N-ZTC based supercapacitor provided a maximum power that is four times larger than that showed by ZTC based supercapacitor (98 and $23 \mathrm{~kW} / \mathrm{kg}$ ), as consequence of the improvement of electrical conductivity produced by N-Q functionalities in N-ZTC electrodes. The 
durability of the capacitors was evaluated by $50000 \mathrm{GCD}$ cycles at $5 \mathrm{~A} / \mathrm{g}$ and $1.2 \mathrm{~V}$. Both capacitors evidenced high capacitance retention after the durability test, but N-ZTC based capacitor showed a most stable performance along cycles, pointing out the stabilizing effect of $\mathrm{N}$ functional groups. These results provide clear evidences of the advantages of doping advanced porous carbon materials with nitrogen functionalities for the improvement of the performance of aqueous based supercapacitors.

Acknowledgments: The authors would like to thank GV and FEDER (PROMETEOII/2014/010), projects CTQ2015-66080-R (MINECO/FEDER) and MAT2016-76595-R (MINECO/FEDER) for financial support. MJML acknowledges Generalitat Valenciana for the financial support through a VALi+d contract (ACIF/2015/374)

\section{References}

[1] F. Béguin, E. Frackowiak, Carbons for Electrochemical Energy Storage and Conversion Systems, $1^{\text {st }}$ ed., Boca Raton, FL: Taylor \& Francis (CRC Press). 2010.

[2] A.G. Pandolfo, A.F. Hollenkamp, Carbon properties and their role in supercapacitors, Journal of Power Sources. 157 (2006) 11-27.

[3] F. Béguin, V. Presser, A. Balducci, E. Frackowiak, Carbons and Electrolytes for Advanced Supercapacitors, Adv. Mater. 26 (2014) 2219-2251.

[4] H. Itoi, H. Nishihara, T. Kogure, T. Kyotani, Three-Dimensionally Arrayed and Mutually Connected 1.2-nm Nanopores for High-Performance Electric Double Layer Capacitor, J. Am. Chem. Soc. 133 (2011) 1165-1167.

[5] H. Nishihara, T. Kyotani, Templated Nanocarbons for Energy Storage, Advanced Materials. 24 (2012) 4473-4498.

[6] H. Itoi, H. Nishihara, T. Ishii, K. Nueangnoraj, R. Berenguer-Betrián, T. Kyotani, Large Pseudocapacitance in Quinone-Functionalized Zeolite-Templated Carbon, Bull Chem Soc Jpn. 87 (2014) 250-257.

[7] H. Itoi, H. Nishihara, T. Kyotani, Effect of Heteroatoms in Ordered Microporous Carbons on Their Electrochemical Capacitance, Langmuir. 32 (2016) 11997-12004. 
[8] O. Ornelas, J.M. Sieben, R. Ruiz-Rosas, E. Morallón, D. Cazorla-Amorós, J. Geng, N. Soin, E. Siores, B.F.G. Johnson, On the origin of the high capacitance of nitrogen-containing carbon nanotubes in acidic and alkaline electrolytes, Chem. Commun. 50 (2014) 11343-11346.

[9] M. Seredych, D. Hulicova-Jurcakova, G.Q. Lu, T.J. Bandosz, Surface functional groups of carbons and the effects of their chemical character, density and accessibility to ions on electrochemical performance, Carbon. 46 (2008) 1475-1488.

[10] D. Hulicova-Jurcakova, M. Seredych, G.Q. Lu, T.J. Bandosz, Combined Effect of Nitrogen- and Oxygen-Containing Functional Groups of Microporous Activated Carbon on its Electrochemical Performance in Supercapacitors, Adv. Funct. Mater. 19 (2009) 438-447.

[11] M.J. Bleda-Martínez, D. Lozano-Castelló, E. Morallón, D. Cazorla-Amorós, A. Linares-Solano, Chemical and electrochemical characterization of porous carbon materials, Carbon. 44 (2006) 2642 2651.

[12] R. Berenguer, R. Ruiz-Rosas, A. Gallardo, D. Cazorla-Amorós, E. Morallón, H. Nishihara, T. Kyotani, J. Rodríguez-Mirasol, T. Cordero, Enhanced electro-oxidation resistance of carbon electrodes induced by phosphorus surface groups, Carbon. 95 (2015) 681-689.

[13] D. Hulicova-jurcakova, A.M. Puziy, O.I. Poddubnaya, F. Suárez-García, J. M. D. Tascón, G. Quing Lu, Highly Stable Performance of Supercapacitors from Phosphorus-Enriched Carbons, J Am Chem Soc 2009; 131(4):5026-7.

[14] D.-W. Wang, F. Li, Z.-G. Chen, G.Q. Lu, H.-M. Cheng, Synthesis and Electrochemical Property of Boron-Doped Mesoporous Carbon in Supercapacitor, Chem. Mater. 20 (2008) 7195-7200.

[15] J.P. Paraknowitsch, A. Thomas, Doping carbons beyond nitrogen: an overview of advanced heteroatom doped carbons with boron, sulphur and phosphorus for energy applications, Energy Environ. Sci. 6 (2013) 2839-2855.

[16] M. Enterría, M.F.R. Pereira, J.I. Martins, J.L. Figueiredo, Hydrothermal functionalization of ordered mesoporous carbons: The effect of boron on supercapacitor performance, Carbon. 95 (2015) 72-83.

[17] M.J. Mostazo-López, R. Ruiz-Rosas, E. Morallón, D. Cazorla-Amorós, Nitrogen doped superporous carbon prepared by a mild method. Enhancement of supercapacitor performance, International Journal of Hydrogen Energy. 41 (2016) 19691-19701. 
[18] M.J. Mostazo-López, R. Ruiz-Rosas, E. Morallón, D. Cazorla-Amorós, Generation of nitrogen functionalities on activated carbons by amidation reactions and Hofmann rearrangement: Chemical and electrochemical characterization, Carbon. 91 (2015) 252-265.

[19] D. Salinas-Torres, S. Shiraishi, E. Morallón, D. Cazorla-Amorós, Improvement of carbon materials performance by nitrogen functional groups in electrochemical capacitors in organic electrolyte at severe conditions, Carbon. 82 (2015) 205-213.

[20] T. Kwon, H. Nishihara, H. Itoi, Q.-H. Yang, T. Kyotani, Enhancement Mechanism of Electrochemical Capacitance in Nitrogen-/Boron-Doped Carbons with Uniform Straight Nanochannels, Langmuir. 25 (2009) 11961-11968.

[21] González-Gaitán C, Ruiz-Rosas R, Morallón E, Cazorla-Amorós D. Electrochemical Methods to Functionalize Carbon Materials. In: V.K. Thakur, M.K. Thakur, editors. Chemical functionalization of carbon nanomaterials, Boca Raton, FL: Taylor \& Francis (CRC Press); 2015: p. 230-261.

[22] C. González-Gaitán, R. Ruiz-Rosas, H. Nishihara, T. Kyotani, E. Morallón, D. CazorlaAmorós, Successful functionalization of superporous zeolite templated carbon using aminobenzene acids and electrochemical methods, Carbon 99 (2016) 157-166.

[23] H. Nishihara, P. Hou, L. Li, M. Ito, M. Uchiyama, T. Kaburagi, et al., High-Pressure Hydrogen Storage in Zeolite-Templated Carbon, J. Phys. Chem. C. 113 (2009) 3189-3196.

[24] P.-X. Hou, H. Orikasa, T. Yamazaki, K. Matsuoka, A. Tomita, N. Setoyama, et al., Synthesis of nitrogen-nontaining microporous carbon with a highly ordered structure and effect of nitrogen doping on $\mathrm{H}_{2} \mathrm{O}$ adsorption, Chem. Mater. 17 (2005) 5187-5193.

[25] Y. Kwon, K. Kim, R. Ryoo, N-doped zeolite-templated carbon as a metal-free electrocatalyst for oxygen reduction, RSC Adv. 6 (2016) 43091-43097.

[26] C.O. Ania, V. Khomenko, E. Raymundo-Piñero, J.B. Parra, F. Béguin, The large electrochemical capacitance of microporous doped carbon obtained by using a zeolite template, Adv. Funct. Mater. 17 (2007) 1828-1836.

[27] K. Nueangnoraj, H. Nishihara, T. Ishii, N. Yamamoto, H. Itoi, R. Berenguer, et al., Pseudocapacitance of zeolite-templated carbon in organic electrolytes, Energy Storage Mater. 1 (2015) 35-41. 
[28] R. Berenguer, H. Nishihara, H. Itoi, T. Ishii, E. Morallón, D. Cazorla-Amorós, et al., Electrochemical generation of oxygen-containing groups in an ordered microporous zeolitetemplated carbon, Carbon 54 (2013) 94-104.

[29] Z. Ma, T. Kyotani, Z. Liu, O. Terasaki, A. Tomita, Very high surface area microporous carbon with a three-dimensional nano-array structure: Synthesis and its molecular structure, Chem. Mater. 13 (2001) 4413-4415.

[30] H. Boehm, Some aspects of the surface chemistry of carbon blacks and other carbons, Carbon 32 (1994) 759-769.

[31] Y. Otake, R.G. Jenkins, Characterization of oxygen-containing surface complexes created on a microporous carbon by air and nitric acid treatment, Carbon 31 (1993) 109-121.

[32] M.C. Román-Martínez, D. Cazorla-Amorós, A. Linares-Solano, C.S.M. de Lecea, TPD and TPR characterization of carbonaceous supports and Pt/C catalysts, Carbon 31 (1993) 895-902.

[33] P. Burg, D. Cagniant, Characterization of carbon surface chemistry, in: L.R. Radovic, editor. Chemistry and Physics of carbons, vol. 30, Boca Raton, FL: Taylor \& Francis (CRC Press); 2008: p. 129-175.

[34] H. Nishihara, Q. Yang, P. Hou, M. Unno, S. Yamauchi, R. Saito, et al., A possible buckybowl-like structure of zeolite templated carbon, Carbon 47 (2009) 1220-1230.

[35] S. Leyva-García, K. Nueangnoraj, D. Lozano-Castelló, H. Nishihara, T. Kyotani, E. Morallón, et al., Characterization of a zeolite-templated carbon by electrochemical quartz crystal microbalance and in situ Raman spectroscopy, Carbon 89 (2015) 63-73.

[36] D. Cazorla-Amorós, D. Lozano-Castelló, E. Morallón, M.J. Bleda-Martínez, A. LinaresSolano, S. Shiraishi, Measuring cycle efficiency and capacitance of chemically activated carbons in propylene carbonate, Carbon 48 (2010) 1451-1456.

[37] O. Barbieri, M. Hahn, A. Herzog, R. Kötz, Capacitance limits of high surface area activated carbons for double layer capacitors, Carbon 43 (2005) 1303-1310.

[38] D. Hulicova-Jurcakova, M. Kodama, S. Shiraishi, H. Hatori, Z.H. Zhu, G.Q. Lu, Nitrogenenriched nonporous carbon electrodes with extraordinary supercapacitance, Adv. Funct. Mater. 19 (2009) 1800-1809. 
[39] S.L. Candelaria, B.B. Garcia, D. Liu, G. Cao, Nitrogen modification of highly porous carbon for improved supercapacitor performance, J. Mater. Chem. 22 (2012) 9884-9889.

[40] M. Kawaguchi, T. Yamanaka, Y. Hayashi, H. Oda, Preparation and capacitive properties of a carbonaceous material containing nitrogen, J. Electrochem. Soc. 157 (2010) A35-A40.

[41] R. Ruiz-Rosas, M.J. Valero-Romero, D. Salinas-Torres, J. Rodríguez-Mirasol, T. Cordero, E. Morallón, et al., Electrochemical performance of hierarchical porous carbon materials obtained from the infiltration of lignin into zeolite templates., ChemSusChem 7 (2014) 1458-1467.

[42] B.E. Conway, Electrochemical supercapacitors: Scientific Fundamentals and Technological Applications. New York: Springer; 1999.

[43] R. Kötz, M. Carlen, Principles and applications of electrochemical capacitors, Electrochim. Acta 45 (2000) 2483-2498.

[44] G. Lota, K. Lota, E. Frackowiak, Nanotubes based composites rich in nitrogen for supercapacitor application, Electrochem. Commun. 9 (2007) 1828-1832.

[45] Y. Zhou, X. Xu, B. Shan, Y. Wen, T. Jiang, J. Lu, et al., Tuning and understanding the supercapacitance of heteroatom-doped graphene, Energy Storage Mater. 1 (2015) 103-111.

[46] V. Strelko, V. Kuts, P. Thrower, On the mechanism of possible influence of heteroatoms of nitrogen, boron and phosphorus in a carbon matrix on the catalytic activity of carbons in electron transfer reactions, Carbon 38 (2000) 1499-1503.

[47] M.J. Bleda-Martínez, J.A. Maciá-Agulló, D. Lozano-Castelló, E. Morallón, D. CazorlaAmorós, A. Linares-Solano, Role of surface chemistry on electric double layer capacitance of carbon materials, Carbon 43 (2005) 2677-2684.

[48] H.A. Andreas, B.E. Conway, Examination of the double-layer capacitance of an high specific-area C-cloth electrode as titrated from acidic to alkaline pHs, Electrochim. Acta 51 (2006) 6510-6520.

[49] K. Nueangnoraj, R. Ruiz-Rosas, H. Nishihara, S. Shiraishi, E. Morallón, D. Cazorla-Amorós, et al., Carbon-carbon asymmetric aqueous capacitor by pseudocapacitive positive and stable negative electrodes, Carbon 67 (2014) 792-794. 
[50] S. Fletcher, V.J. Black, I. Kirkpatrick, A universal equivalent circuit for carbon-based supercapacitors, J. Solid State Electrochem. 18 (2014) 1377-1387.

[51] S.L. Candelaria, Y. Shao, W. Zhou, X. Li, J. Xiao, J. Zhang, et al., Nanostructured carbon for energy storage and conversion, Nano Energy 1 (2012) 195-220.

[52] Y. Zhai, Y. Dou, D. Zhao, P.F. Fulvio, R.T. Mayes, S. Dai, Carbon materials for chemical capacitive energy storage, Adv. Mater. 23 (2011) 4828-4850.

[53] T. Le, Y. Yang, Z. Huang, F. Kang, Preparation of microporous carbon nanofibers from polyimide by using polyvinyl pyrrolidone as template and their capacitive performance, J. Power Sources 278 (2015) 683-692.

[54] D. Salinas-Torres, R. Ruiz-Rosas, M.J. Valero-Romero, J. Rodríguez-Mirasol, T. Cordero, E. Morallón, et al., Asymmetric capacitors using lignin-based hierarchical porous carbons, J. Power Sources 326 (2016) 641-651.

[55] D.N. Futaba, K. Hata, T. Yamada, T. Hiraoka, Y. Hayamizu, Y. Kakudate, et al., Shapeengineerable and highly densely packed single-walled carbon nanotubes and their application as super-capacitor electrodes, Nat. Mater. 5 (2006) 987-994.

[56] G.A. Ferrero, A.B. Fuertes, M. Sevilla, N-doped porous carbon capsules with tunable porosity for high-performance supercapacitors, J. Mater. Chem. A 3 (2015) 2914-2923. 